\title{
ANNUAL CYCLES IN THE INCIDENCE OF B CHROMOSOMES IN THE GRASSHOPPER MYRMELEOTETTIX MACULATUS (ACRIDIDAE: ORTHOPTERA)
}

\author{
PETER M. ROBINSON* and GODFREY M. HEWITT \\ School of Biological Sciences, University of Eost Anglia, Norwich NR4 7TJ, U.K.
}

Received 29.i.76

\section{SUMMARY}

The frequency of B chromosomes has been determined for several stages in the grasshopper life-cycle, over three generations, in three natural populations spanning a steep cline in East Anglia. This has revealed a rise in B frequency in prediapause eggs, a fall during the winter in the embryos and a rise in the early nymphs in certain situations. The causes of these effects are discussed and also their importance, along with meiotic drive, in maintaining an environmentally correlated cline of stable adult B frequencies.

\section{Introduction}

B CHROMOSOMEs occur in many animal and plant species, but in only a handful of cases is there even fragmentary information on their possible adaptive roles in natural populations (Jones, 1975). The properties of $\mathrm{B}$ chromosomes vary considerably between species, and it seemed to us that this situation is best approached by a study in depth of the various components of one particular system.

In Southern Britain the grasshopper Myrmeleotettix maculatus is polymorphic for a large metacentric B chromosome, to which no obvious morphological effects have so far been attributed. However, it does increase chiasma frequency and variance (John and Hewitt, 1965a, b; Barker, 1966; Hewitt and John, 1967, 1970). This B chromosome is mitotically stable, and crosses reveal some form of " meiotic drive" when it is transmitted through the female and " meiotic drag" through the male (Hewitt, 1973a). Furthermore, populations differ in the degree of drive and drag they display (Hewitt, $1973 b, c)$. The distribution of the $\mathrm{B}$ chromosome polymorphism is broadly associated with the warmer and drier conditions that generally favour Acridids (Dempster, 1963), and detailed studies of frequency clines in West Wales and East Anglia show a close correlation between the B chromosome and the microclimate of the grasshopper (Hewitt and Brown, 1970; Hewitt and Ruscoe, 1971; Robinson, 1973).

The adult frequency of $B$ chromosomes in many of these populations has been stable for several years and it seems necessary to postulate certain selective forces to account for this. The clines are also most readily explained by differential environmental selection. Therefore, we chose to determine the frequency of this polymorphism through the various phases of the lifecycle, coupled with studies of the population ecology, and thereby reveal the degree of selection, the stage at which it operates and something of its nature.

* Present address: Social Studies, Teeside Polytechnic, Middlesborough, TS1 3BA, U.K. 


\section{Materials and methods}

The adults of $M$. maculatus lay their egg pods during the summer months in small patches of bare earth in the mosaic vegetation which the species prefers. The eggs, of which there are 4-5 in a pod, undergo a short period of development before entering diapause, when development ceases, and this is the form in which the species overwinters, as all the adults die off in the autumn. Embryonic development recommences in the spring and hatching of the immature nymphal forms begins in May. There are four nymphal instars, each with a duration of about 10-14 days. The adults emerge towards the end of June.

The East Anglian cline is an excellent situation for this study, as a large number of populations are available fairly close together and many have now been studied for a number of years (e.g. Hewitt and Brown, 1970; Robinson, 1973). Furthermore, valuable background work on the ecology and microenvironment of the species in East Anglia has previously been done by Brown (1968) and Ruscoe (1970). Three populations were chosen for the present study (fig. 1); Wortham, which has a very low frequency of B chromosomes (around 5 per cent), Rushford with an intermediate level ( 25 per cent), and finally Foxhole, which has a high frequency of supernumeraries (50 per cent). Ideally the populations chosen for study should have been of similar size, but this was not possible. Wortham is the smallest of the populations and Rushford the largest; peak adult numbers during this three-year study (1970-1973) were approximately as follows: Wortham 800, Foxhole 2500 and Rushford 6000.

\section{(i) The egg stage}

For field sampling of the egg populations, recognition of likely oviposition sites was a pre-requisite, and this became possible following three lines of enquiry. First, the ecological requirements of the laying females, the eggs and the newly hatched nymphs were considered. Then, exploratory field sampling using transects was carried out. Finally, the results of laboratory investigations of the oviposition behaviour of British Acridids (Moriarty, 1969; Kelly-Stebbings and Hewitt, 1972) were considered with these two. This synthesis revealed the oviposition site to be a patch of bare earth, well drained and rarely greater than $0.25 \mathrm{~m}^{2}$ in area. The earth is not loose, reducing the risk of exposure of pods by erosion. Grass and other vegetation is always close at hand, providing food and shelter for the adults and the newly hatched nymphs.

Random samples of $M$. maculatus oviposition sites were taken and the egg pods revealed by sieving. Samples were taken on each of two occasions in the 1970-71 and 1971-72 generations in the autumn after oviposition was complete, (October-November) and again in the spring after the completion of diapause and prior to hatching (March-April). The populations were sampled as near to the same time as possible for the sake of comparison, but there was some variation because of the time-consuming nature of the sampling technique and its reliance on fine weather. Additional samples were taken during the intervening winter period in the 1972-73 generation in an attempt to define more accurately the variation observed in the two previous years. 
Egg pods from the autumn samples were stored in petri dishes of sterile, moist sand (10 per cent water by weight) for eight weeks at $4^{\circ} \mathrm{C}$ to break the diapause. At the end of this period the eggs were removed from the pods, the embryos dissected out, incubated in a solution of 0.05 per cent colchicine in hypotonic saline for one hour at $35^{\circ} \mathrm{C}$, and then fixed in $3: 1$ alcohol:acetic acid. The treatment of egg pods obtained from the winter and spring samples was essentially the same except for appropriate variation in the time of treatment at $4^{\circ} \mathrm{C}$ to compensate for the increased time these eggs had been

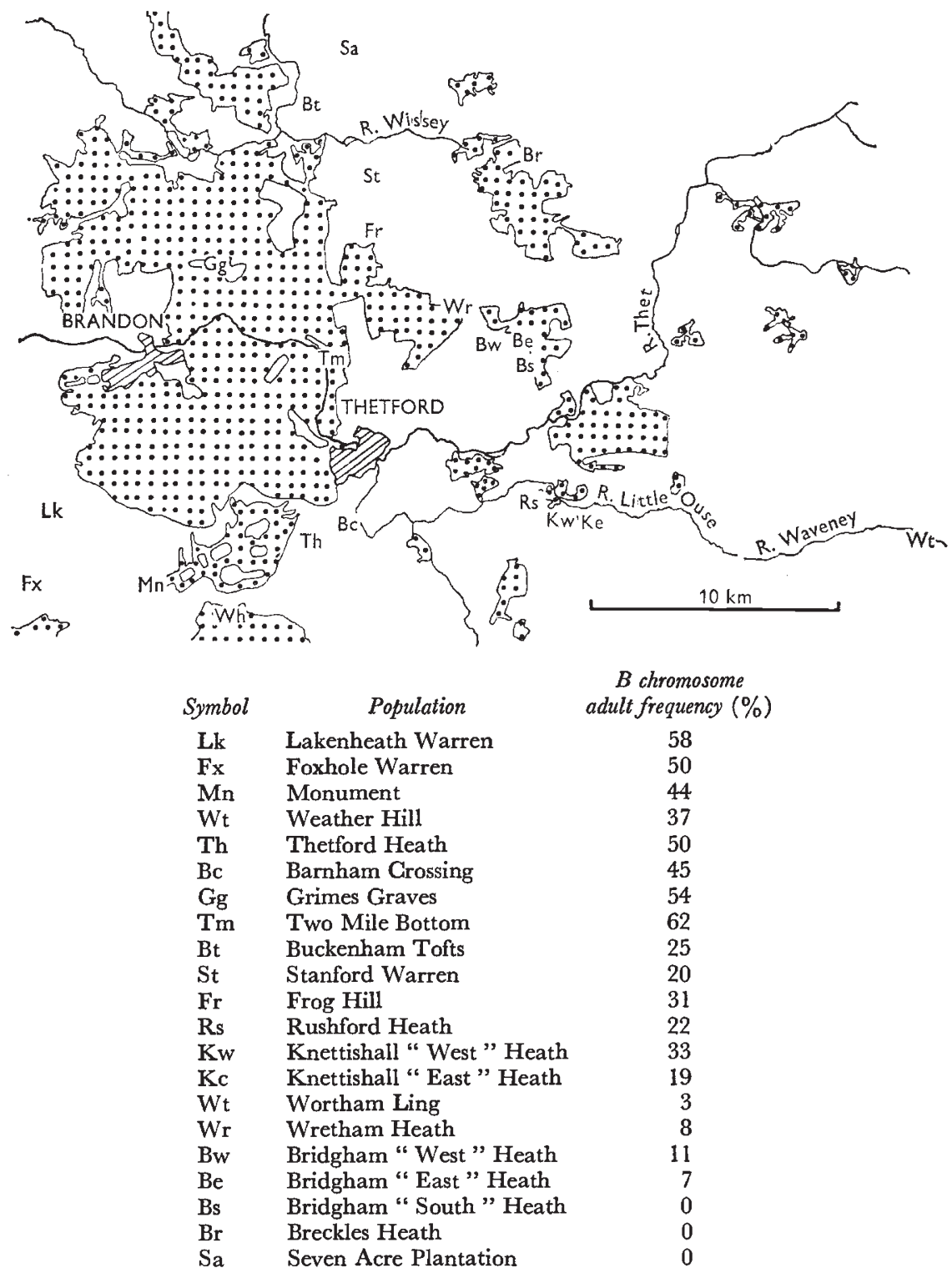

Fig. 1.-A map of the East Anglian cline in B chromosome frequency. The stippled areas represent the larger woods and the two towns are diagonally lined. 
in the field; thus the " winter " eggs were exposed to $4^{\circ} \mathrm{C}$ for about $4-5$ weeks and the "spring" ones were given no cold treatment at all. The treatments outlined above produce embryos which are at about stage 22 on Uvarov's (1966) scale of acridid development, and have a sufficiently high mitotic index for successful karyotyping using the usual lacto-propionic orcein squash techniques.

A possible objection to this method is that differential mortality of B or $\mathrm{OB}$ embryos might occur during the long period of cold treatment and subsequent incubation, thereby invalidating the obtained estimate of $\mathrm{B}$ chromosome frequency as an accurate reflection of the situation existing in the field populations at the time of sampling. However, when the same technique is applied to batches of eggs conceived and laid in the laboratory, over 95 per cent of the eggs which do develop to embryos can be karyotyped. Furthermore, they include many which carry numerical chromosome mutations of varying degrees of severity, and if these survive the technique, then it suggests that it is not differentially killing $0 \mathrm{~B}$ - or B-containing embryos.

\section{(ii) The nymphal instars}

Random samples of the nymphal populations were taken using nets and pooters during the spring of 1971. Karyotyping them was extremely difficult and therefore samples were not taken in the following years. The first and second instar nymphs of this species are so small that it is virtually impossible to remove and fix any tissues for conventional squash techniques of karyotyping. Even the larger third and fourth instar animals of this species are not so large that tissues such as the gonads and gut caecae, which usually yield suitable materials for karyotyping, can be removed effectively.

The technique adopted for all instars was to slit each nymph along the ventral surface, incubate it in a 0.05 per cent solution of colchicine in hypotonic insect saline and then fix it whole in the $3: 1$ alcohol:acetic acid. After 2-3 weeks the integument can be stripped away and lacto-propionic orcein squashes made of the remaining material. This material has a very low mitotic index; karyotyping was therefore not always successful, and although three successive samples were taken at each population in 1971 , the results have been pooled to give a single estimate of nymphal B chromosome frequency for each population.

\section{(iii) The adults}

Random samples of adult males were collected from the three populations on a number of occasions in each generation. The testes were dissected out, fixed in 3:1 alcohol:acetic acid and lacto-propionic orcein squashes made of this material. Samples of females were not taken as no sex differences have been observed in the frequency of $\mathrm{B}$ chromosomes in populations of $M$. maculatus (Hewitt and John, 1967). Furthermore, sampling females would involve a considerable loss in a population's reproductive capacity, which would be inadvisable when studying an equilibrium situation over several years.

\section{Results}

The results are summarised in tables $1,2,3$ and 4 and displayed graphically in fig. 2. Despite the sizes of the errors on the various estimates of the 


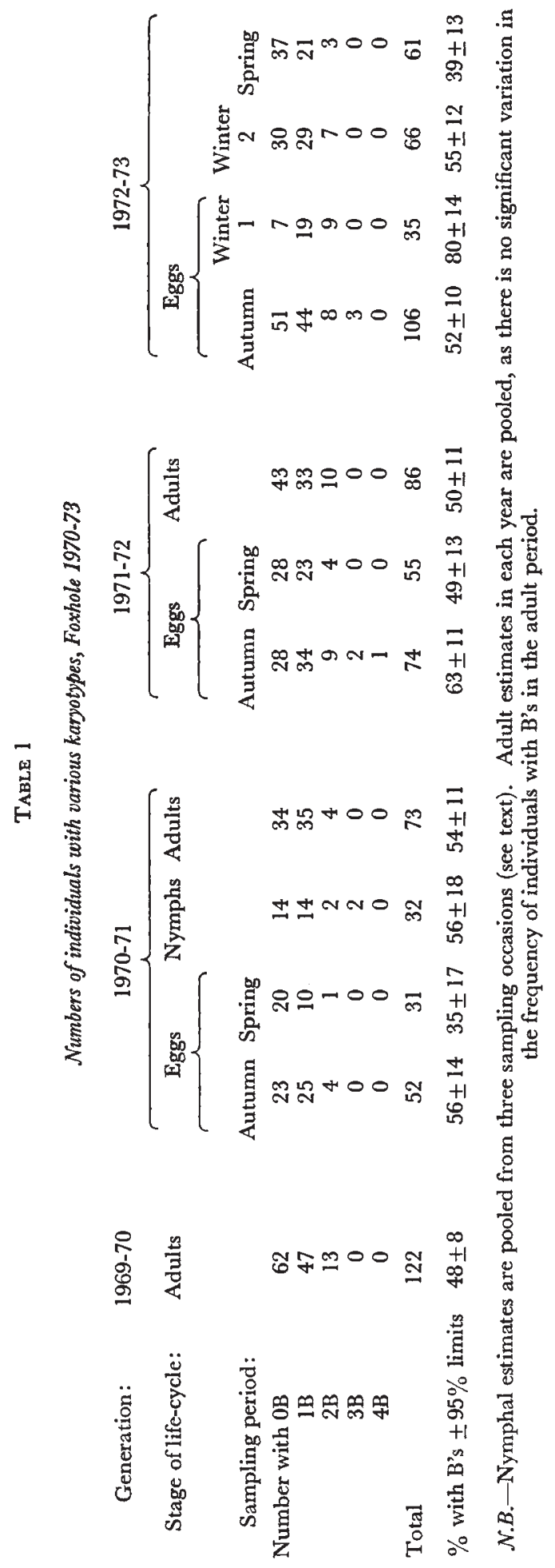




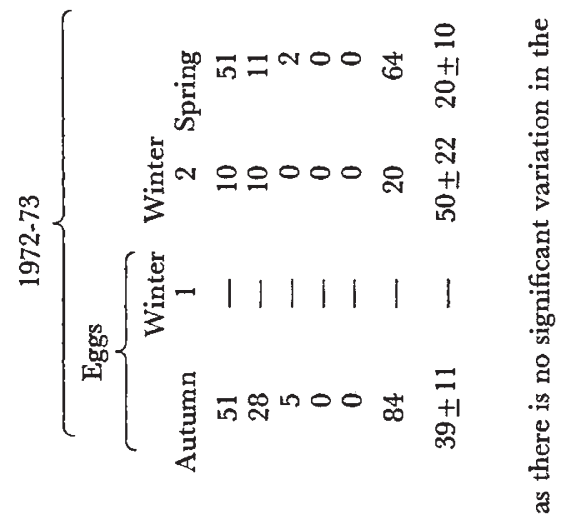

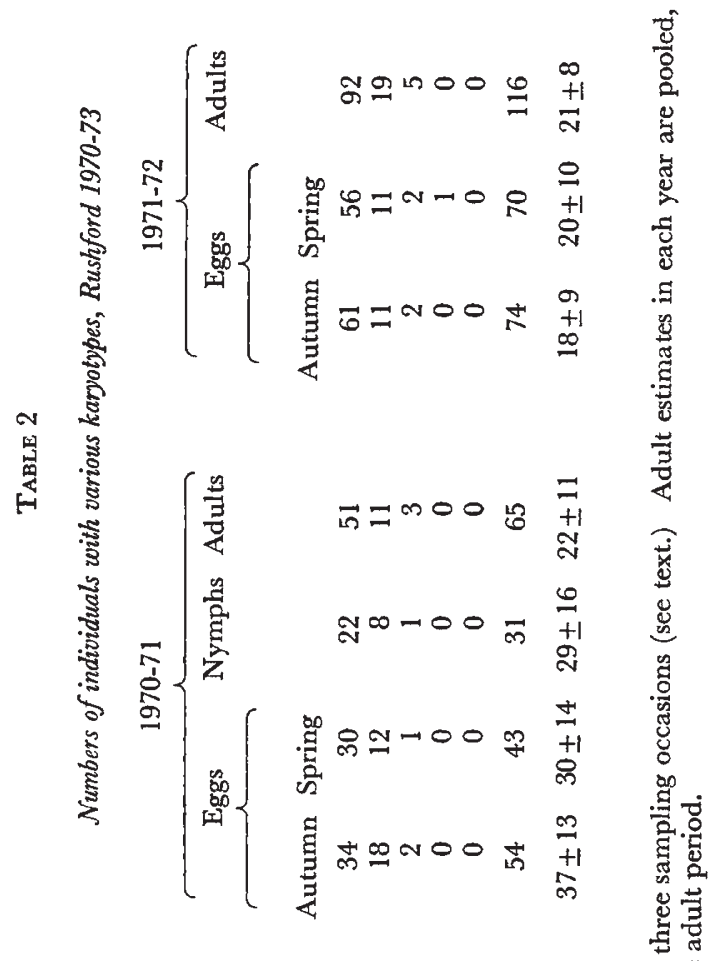

$$
\begin{aligned}
& \text { 号 菊 } \\
& \text { ㄷํㅇ현 } \\
& \text { 南 } \\
& \text { 专运 }
\end{aligned}
$$

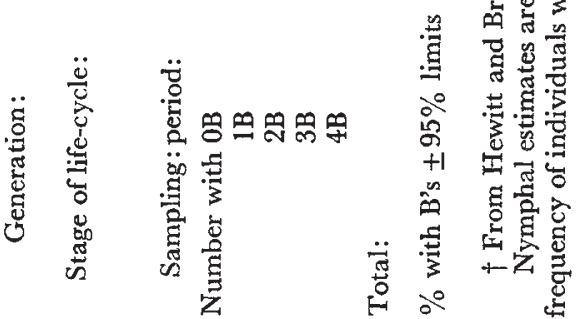




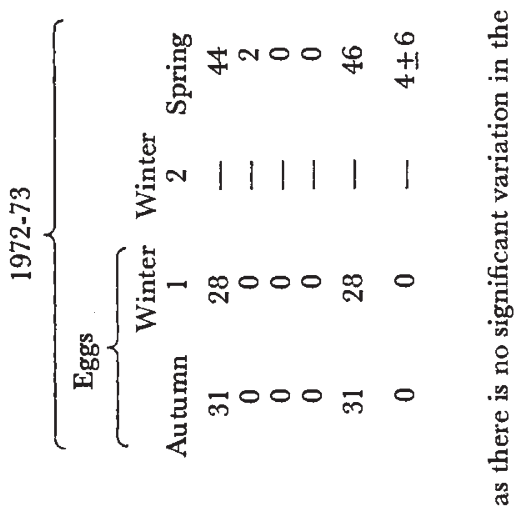

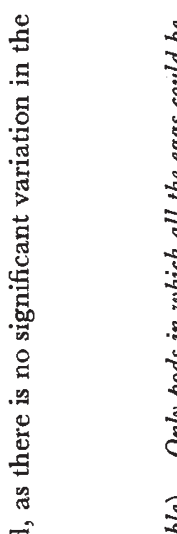

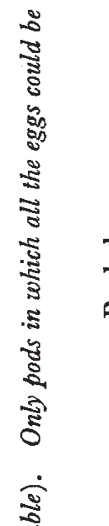

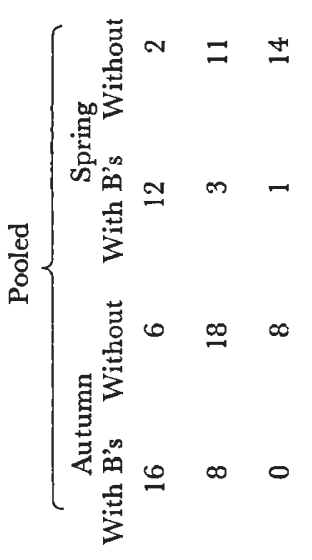

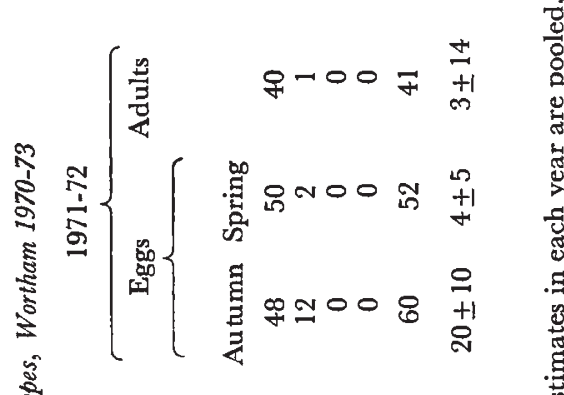

胥

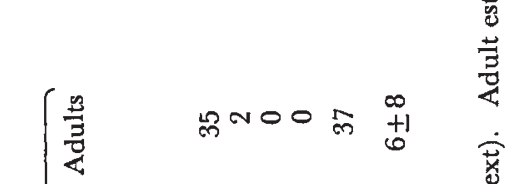

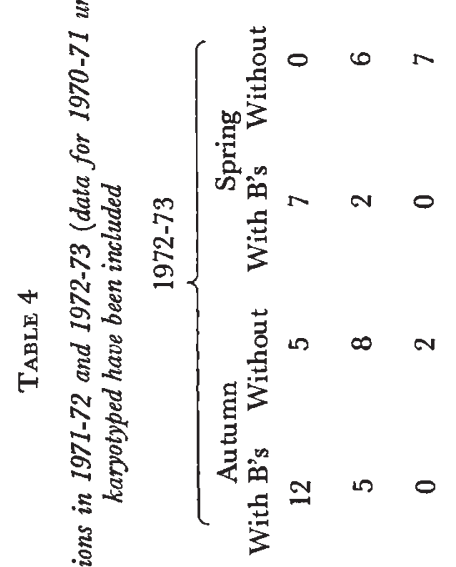

乼综

m०00 ले 0 造

बू

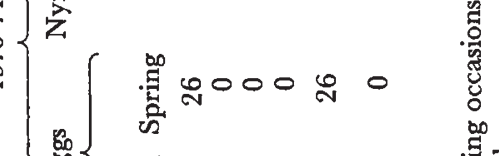

है

(

递泀

के छ

웅 莒

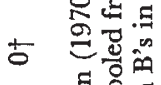

雾运

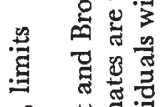

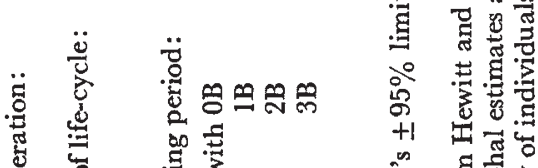

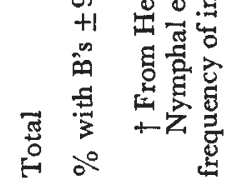

36/3-H

究

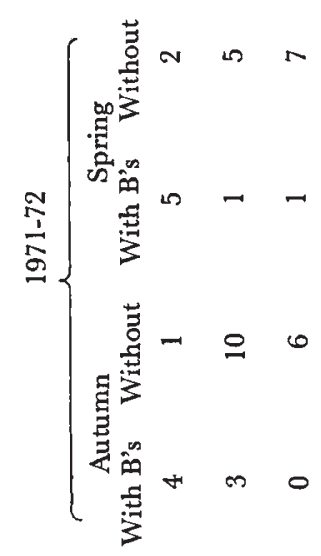

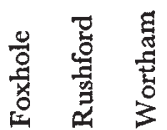


frequency of individuals with B chromosomes, owing to the necessarily small sample sizes, the results nevertheless reveal a striking and consistent pattern of variation within a generation. The frequency of individuals carrying $\mathrm{B}$ chromosomes in the autumn egg samples is greater than in the preceding adult generation which produced those eggs, and this is followed by a decrease

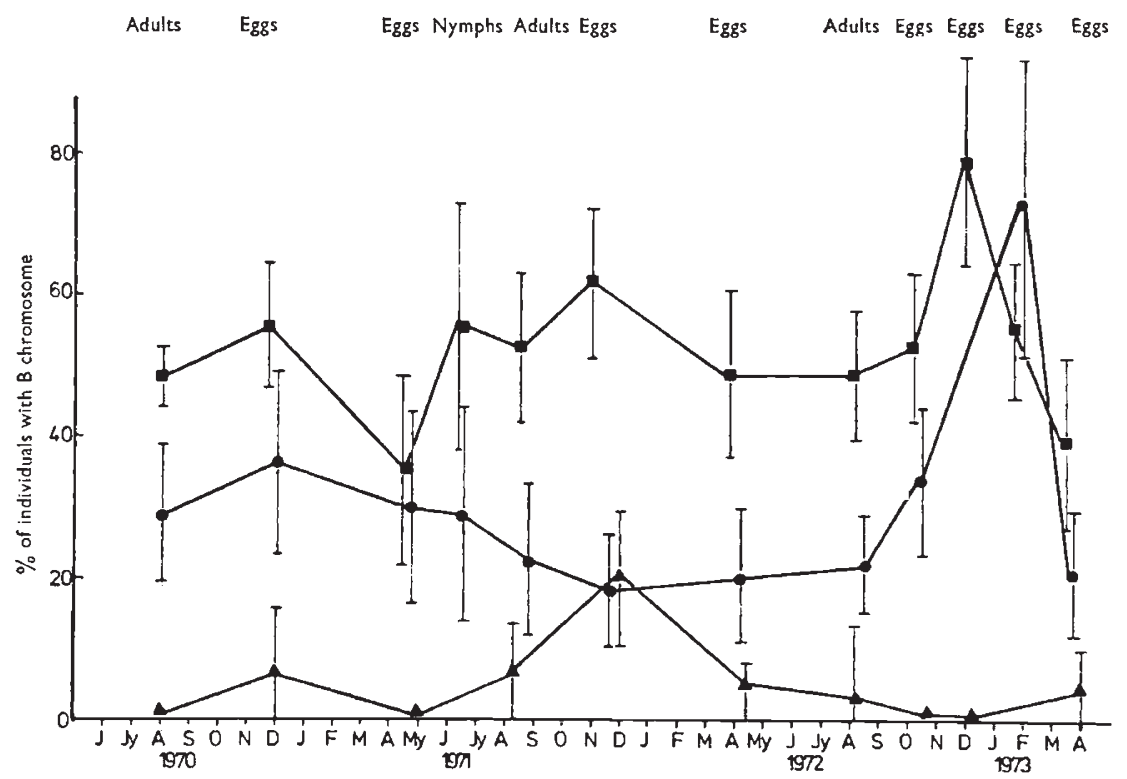

Fig. 2.-Variation in the percentage of individuals with $B$ chromosomes through the stages of the life-cycle, 1970-1973, in the three populations: Foxhole at the top, Rushford in the middle and Wortham at the bottom. The bars show 95 per cent confidence limits.

in B chromosome frequency during the winter up to the time of the spring egg samples. At Rushford and Wortham, which have intermediate and low $\mathrm{B}$ chromosome frequencies respectively, this winter decrease is to a level about that of the subsequent adult population, whereas at Foxhole, which has a higher incidence of $\mathrm{B}$ chromosomes, the winter decline is to a lower level. This difference between the populations is shown markedly in the average change in B chromosome frequency shown by each population over the three years (fig. 3). Differences in the incidence of $\mathrm{B}$ chromosomes between an adult generation and the subsequent autumn egg sample are only significant at Rushford in 1972-1973 $\left(\chi^{2}, \mathrm{P}=0.001\right)$ and in Wortham in 1971-1972 $\left(\chi^{2}, \mathrm{P}=0.02-0 \cdot 05\right)$, although if the results for the three years are pooled for each population, then in all three the change between adults and autumn egg samples is significant $\left(\chi^{2}, \mathrm{P}=0.02-0.05\right.$ (Foxhole and Wortham) and $\mathrm{P}=0.01-0.02$ (Rushford)).

Although the pattern of change during the egg stage is similar in all three populations over three years, Rushford in 1971-1972 and Wortham 19721973 are exceptions to this, showing no apparent change in B chromosome frequency. The explanation of this is uncertain; at Wortham it may be a simple one of sampling error resulting from that population's very low overall level of $\mathrm{B}$ chromosomes and the small number of samples. This explanation is less plausible in the case of the Rushford result. It was thought that 
between 1970 and 1971 the microclimate of this habitat might have become less favourable to grasshoppers carrying B chromosomes. This argument became untenable when the fluctuation in the egg stage re-appeared in 19721973 without any habitat change.

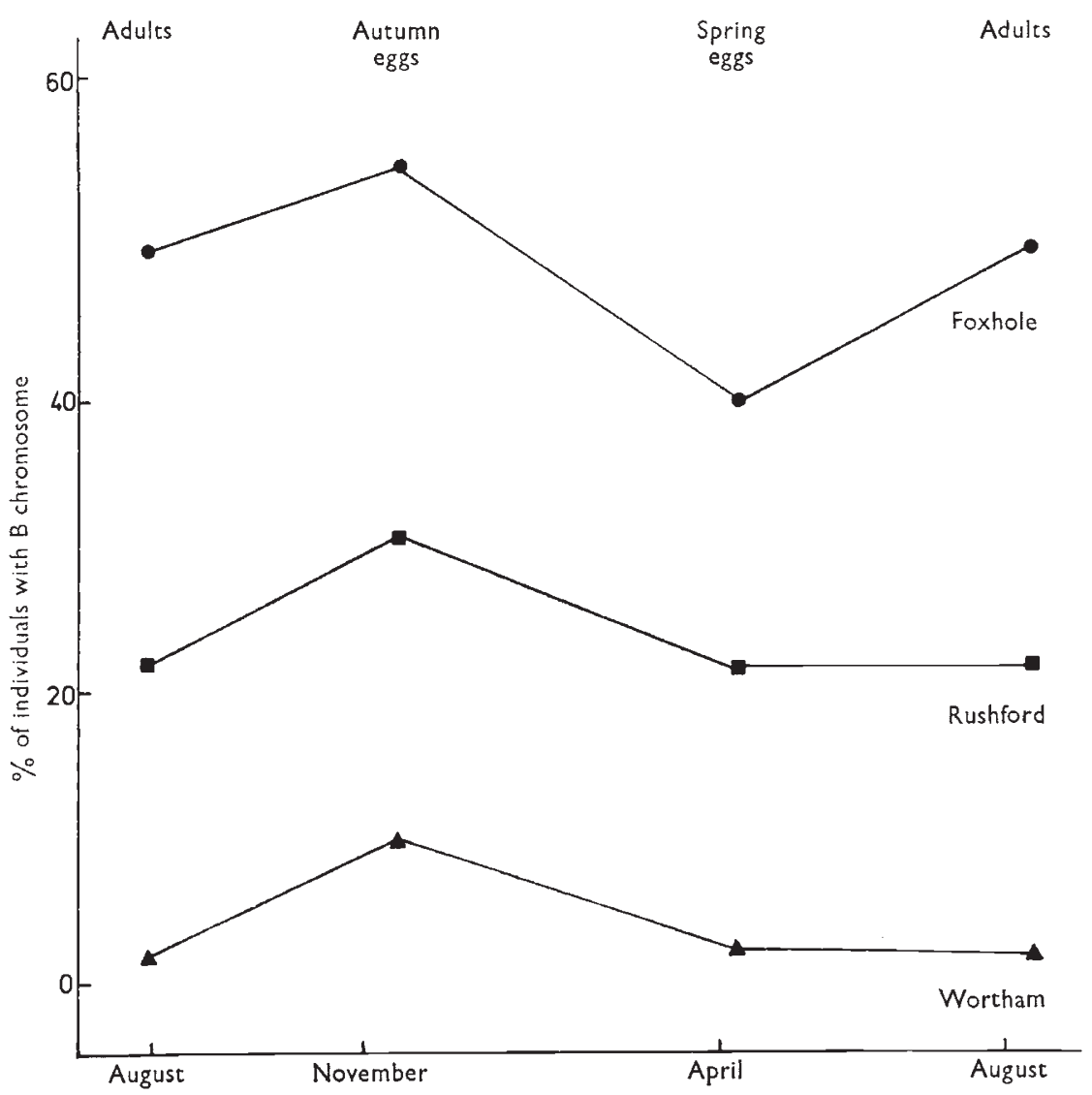

Fig. 3.-Mean fluctuation in the frequency of individuals with $B$ chromosomes at the three populations, Foxhole, Rushford and Wortham; 1970-1973.

Owing to the considerable inefficiency of the available techniques, nymphal karyotyping was only attempted in 1971, and the results of several samples at each population have had to be pooled. On the basis of these somewhat imprecise estimates of the nymphal B chromosomes, there appears to be no significant difference in 1971 between the level of the supernumerary in the spring egg samples and its level in the nymphs $\left(\chi^{2}\right.$, Foxhole $\mathrm{P}=0 \cdot 1$, Rushford $\mathrm{P}=0 \cdot 7-0 \cdot 8$, Wortham $\mathrm{P}=0.99$ ). Even so, at Foxhole there is a marked rise in $\mathrm{B}$ chromosome frequency between the spring eggs and the nymphs, and although this is not statistically significant, it is noteworthy that in the subsequent two years of study at this population the incidence of B chromosomes in the spring eggs was also below that in the first adult samples, suggesting that the B chromosome level may not be stable in this period of the life-cycle. It is hoped that more efficient methods of 
karyotyping the very small nymphs may be developed to enable a more thorough investigation of this area.

The results obtained in this study show no significant variation either within or between the adult generations. This confirms that the adult B chromosome frequency in these populations is stable from year to year, as previously shown for both the Welsh and East Anglian clines, but the sample sizes may not be sufficiently sensitive to detect small changes in B chromosome frequency.

\section{Discussion}

The above data clearly demonstrate that marked changes occur in the frequency of B chromosomes at three particular stages in the grasshoppers' life-cycle. Firstly, there is a significant rise in B chromosome frequency of about 6 per cent on average between the adult population and the autumn egg sampled from the field. Using laboratory crosses of insects sampled from the field, the degree of "female drive" and " male drag" for the B chromosomes has been determined for the Foxhole population (Hewitt, 1973b). These two phenomena appear to be roughly in balance with possibly a small loss of B chromosomes ( $\sim 5$ per cent) between the adult frequency and that of the zygotes they produce. Thus the actual rise in B frequency in the egg between oviposition and autumn sampling could be in the region of 11 per cent. This difference in the observed B frequency and that predicted could be due to non-random mating or differential fecundity of the different karyotypes, but there is no clear evidence for either of these from our investigations to date (Hewitt, 1973b, c; Robinson, 1973). Both require more detailed investigation. However, the most obvious possible cause is differential selection for B-containing embryos, and this raises a number of considerations on the nature of any such selection.

The bulk of the eggs are laid during August, when adult numbers are highest and when they experience high soil temperatures and low levels of soil moisture. Salt (1952) and Pickford (1966) have demonstrated that such conditions can adversely affect egg survival in Acridids. Soil temperature is a component of the microclimate, and the latter has been shown to vary across the East Anglian cline (Robinson, 1973), and therefore if it were the selective agent then one might expect this to be reflected in the magnitude of the change in B chromosome frequency between oviposition and the autumn. The results show that the absolute change in $\mathrm{B}$ chromosome levels is about the same in all three populations studied. However, the three populations have different equilibrium $B$ frequencies, and therefore the proportional rise in B frequency is greater for the lower adult B frequency. Thus the greatest proportional rise is in the Wortham population, with Rushford next and Foxhole with the lowest; this is inversely related to the soil temperature in these locations at that time of year. Rainfall shows little variation across the East Anglian cline, but the soil is sandy at Foxhole whilst Rushford and Wortham have increasingly heavy soil types. Consequently the early egg's environment is on average much drier at Foxhole than at Rushford or than at Wortham, and the variation in soil temperature acts to augment these differences. This suggests that hot dry soils may be less favourable to Bcontaining embryos than cooler damper ones; but this in itself does not adequately explain the general rise in B frequency in the early egg stage and it 
does not help to account for the correlation of the distribution of B chromosomes with warmer drier summer conditions within Britain. Acridid eggs are very resistant to environmental stresses once the diapause starts (Dempster, 1963); thus any selection between oviposition and the autumn samples could be determined by the speed with which embryos of different karyotypes reach diapause, and not depend on the physiological tolerance of the prediapause embryos themselves. It is of course possible that two forces are operating in this period, one increasing and one decreasing B frequency. Whilst at present it is not clear what is causing this significant rise in B chromosome frequency in the early eggs, nevertheless we have located it to a short phase in the life-cycle and it is amenable to experimental investigation.

The second period in which the results show a significant change in $\mathrm{B}$ frequency is between the autumn and spring samples, when the frequency declines in all populations. This is interpreted as selection now acting against those embryos carrying B chromosomes, which is further evidenced by the marked decrease over this period of the number of embryos with two or more B chromosomes. The reduced frequency of B-containing embryos in the spring samples does not appear to be the result of biased sampling, as there is no difference in the proportion of $\mathrm{B}$ chromosome containing pods in the autumn and spring samples (see table 4).

The average magnitude of this change in $\mathrm{B}$ chromosome frequency during the winter appears to be different at each population. At Foxhole it drops 17 per cent from 57.5 per cent, a 30 per cent fall proportionately; at Rushford it drops 8.5 per cent from 31.5 per cent, a 27 per cent fall proportionately, whilst at Wortham it drops 3.5 per cent from 9.0 per cent, a 61 per cent fall proportionately. Thus the proportionate selection against $\mathrm{B}$ chromosome containing individuals is about the same in Foxhole and Rushford but appears to be about double in Wortham. Now Wortham has a very low $B$ frequency and this makes the estimated fall of 61 per cent less accurate than the others, but it does suggest that this type of selection may be important in determining that certain populations have low B chromosome frequencies.

It is most probable that this period involves a different selective agent to that which is operating between oviposition and the autumn sampling. The parallel study of the population ecology of these populations indicates that the predation of egg pods is a significant mortality factor during the winter (Robinson, 1973). However, as the magnitude of predation varies considerably between years, and as predators are most likely to find egg pods by random searching of oviposition sites, predation seems an unlikely candidate for a selective agent.

Of the other mortality factors known to operate on acridid eggs, little is known of the effects of disease, but it becomes important only infrequently (see Dempster, 1963), and as parasitism was never encountered in any of the eggs, then climate in some form appears the most likely of the known mortality factors which might act selectively.

Although climate is known to influence survival of Acridid eggs (Dempster, 1963; Pickford, 1970), most evidence indicates that eggs in diapause are quite resistant to environmental stresses. It is possible that short periods of mild weather during the winter could interrupt diapause and make the eggs susceptible. Egg survival in the laboratory is affected by soil moisture content, and we have already noted that Wortham has a heavier 
soil than Rushford and Foxhole. The extra samples taken from Foxhole during the winter of 1972-1973 indicate that the loss of B-containing embryos occurs continuously through this period.

Thirdly, the data available on the nymphal stages do not allow us to draw such firm conclusions about the dynamics of the B chromosome during this period of the life-cycle. At Rushford and Wortham the frequency of B chromosomes in the spring egg samples is very close to the stable adult level, suggesting that little net change is taking place in the nymphal instars. Foxhole alone appears to be subject to a fluctuation in B chromosome levels at this time, as the B chromosome frequency in the spring egg samples consistently falls to a level below that of the stable adult level. The single estimate for the nymphal period offers some suggestion that the increase between the spring eggs and the adults is occurring prior to, during, or just after hatching. Furthermore, all three populations exhibit similar nymphal mortality rates (Robinson, 1973). This post diapause rise in B frequency at Foxhole could be particularly significant since this population has a high equilibrium frequency of B chromosomes.

There is no evidence of any great differences in B chromosome frequency or mortality rates between the three populations during the adult period. The sample size employed would not necessarily detect changes of about less than 10 per cent, but it was important not to perturb the B chromosome equilibrium by oversampling. Consequently, selection for or against the survival of B-containing older nymphs and adults does not appear to be a major factor in the dynamics of this chromosome polymorphism.

The $\mathrm{B}$ chromosome frequency in the adults is strongly correlated with the macro- and micro-environment of the population, particularly in the clines studied in East Anglia and West Wales (Hewitt and Brown, 1970; Hewitt and Ruscoe, 1971). This frequency is also strikingly stable, in some cases for up to nine years now. The demonstration here of at least three changes in $B$ frequency in the egg stage through to early nymphs, but no temporal adult variation, indicates that any casual relationship between environment and $B$ frequency is determined largely through effects on the egg stage. The equilibrium frequencies are thus maintained by (1) the resultant of male "drag " and female "drive" in transmission, (2) the rise in frequency in the pre-diapause eggs (which may also have more than one component), (3) the selection against B embryos during the winter and (4) a rise in $\mathrm{B}$ frequency in post-diapause eggs or early nymphs in some situations (i.e. Foxhole). The warm dry summer conditions could affect any of these, except the winter selection, to produce the positive correlation with B frequency; with the identification of these stages, this is open to laboratory investigation. The operation of several counterposed factors will tend to maintain the constancy of B chromosome frequency over several years, and the observation of increased selection in the winter against individuals with two or more B chromosomes demonstrates a frequency-dependent element which will stabilise the equilibrium. Furthermore, it seems likely that more forces are operating with lesser effect than those we have identified.

In a general context this demonstration of three periods of differential selection for and against B chromosomes clearly refutes the suggestions that B chromosomes are "nuclear parasites", with no beneficial effects and maintained only by their accumulation mechanisms (Östergren, 1945; Rhoades and Dempsey, 1972). Obviously the accumulation mechanism is 
an important component in Myrmeleotettix maculatus, but it varies between populations and is not apparently the overriding factor in the Foxhole population at least. However, accumulation mechanisms may well be a sine qua non in the establishment of new B chromosomes (Hewitt, 1973c). Recent experiments on Secale cereale and Lolium perenne have shown that plants containing $\mathrm{B}$ chromosomes are at an advantage under certain sowing densities and selected against under others; and this differs between the two species (Rees and Ayonoadu, 1973; Rees and Hutchinson, 1973; Hutchinson, 1975). Together these works demonstrate the complexity of the factors involved in these polymorphisms and the need for detailed population studies to elucidate them, since a range of selective changes in B frequency within a generation may be masked by a stable adult frequency.

Acknowledgments.-This work was financed in part by a grant from the Science Research Council (G.H.); one of us (P.R.) received a N.E.R.C. studentship; Mrs Janet Robinson's assistance was invaluable, and we are most grateful for all this support.

\section{REFERENCES}

BARKER, J. F. 1966. Climatological distribution of a grasshopper supernumerary chromosome. Evolution, 20, 665-667.

BRown, F. M. 1968. Population cytogenetics of Myrmeleotettix maculatus. M.Sc. thesis, University of East Anglia.

DEMPSTER, J. P. 1963. The population dynamics of grasshoppers and locusts. Biol. Rev., $38,490-529$.

HEWITT, G. M. 1973a. Variable transmission rates of a B-chromosome in Myrmeleotettix maculatus (Thunb.) (Acrididae : Orthoptera). Chromosoma, 40, 83-106.

HEWITt, G. M. 1973b. Evolution and maintenance of B-chromosomes. Chromosomes Today, 4, 351-369.

HEWITT, G. M. 1973c. The integration of supernumerary chromosomes into the orthopteran genome. Cold Spring Harbor Symp. Quant. Biol., 38, 183-194.

HEWITT, G. M., AND BROWN, F. M. 1970. The B-chromosome system of Myrmeleotettix maculatus. V. A steep cline in East Anglia. Heredity, 25, 363-371.

HEWITT, G. M., AND JOHN, B. 1967. The B-chromosome system of Myrmeleotettix maculatus (Thunb.). III. The statistics. Chromosoma, 21, 140-162.

HEWITT, G. M., AND JoHN, B. 1970. The B-chromosome system of Myrmeleotettix maculatus (Thunb.). IV. The dynamics. Evolution, 24, 169-180.

HEWITT, G. M., AND RUSCOE, C. N. E. 1971. Changes in microclimate correlated with a cline for B-chromosomes in the grasshopper Myrmeleotettix maculatus. 7. Anim. Ecol., 40, $753-765$.

hutchinson, J. 1975. Selection of B-chromosomes in Secale cereale and Lolium perenne. Heredity, 34, 39-52.

JoHn, B., AND HEWITT, G. M. 1965a. The B-chromosome system of Myrmeleotettix maculatus (Thunb.). I. The mechanics. Chromosoma, 16, 548-578.

John, B., AND heWrTt, G. м. 1965b. The B-chromosome system of Myrmeleotettix maculatus (Thunb.). II. The statistics. Chromosoma, 17, 121-138.

JONES, R. N. 1975. B-chromosome systems in flowering plants and animal species. Int. Rev. Cytol., 40, 1-100.

KELLY-STEBBINGS, A., AND HEWITT, G. M. 1972. The laboratory breeding of British grasshoppers. Acrida, 1, 233-275.

MORIARTY, F. 1969. The laboratory breeding and embryonic development of Chorthippus brunneus Thunberg (Orthoptera : Acrididae). Proc. R. Ent. Soc. Lond. (A), 44, 25-34.

östergren, G. 1945. Parasitic nature of extra fragment chromosomes. Bot. Not. Lund., 2, 157-163.

PICKFORD, R. 1966. The influence of date of oviposition and climatic conditions on hatching of Camnula pelucida (Orthoptera). Can. Entom., 98, 1145-1159.

PICKFORD, R. 1970. The effects of climatic factors on egg survival and fecundity in grasshoppers. Proc. Int. Study Conf. Current and Future Problems in Acridology (London), 257-260. 
REes, h., AND AyonoAdu, u. 1973. B-chromosome selection in rye. Theoret. Appl. Genet., 43, 162-166.

REEs, H., AND hutchinson, J. 1973. Nuclear DNA variation due to B-chromosomes. Cold Spr. Harb. Symp. Quant. Biol., 38, 175-182.

RHOADES, M. M., AND DEMPSEY, E. 1972. On the mechanism of chromatin loss induced by the B-chromosomes of maize. Genetics, 71, 73-96.

ROBINSON, P. M. 1973. The ecological genetics of the polymorphism for B-chromosomes in the grasshopper Myrmeleotettix maculatus (Thunb.) (Acrididae : Orthoptera). Ph.D. thesis, University of East Anglia.

RUSCOE, C. N. E. 1970. A comparative study of the humidity responses of ecologically distinct species of British grasshoppers. Ph.D. thesis, University of East Anglia.

SALT, R. W. 1952. Some aspects of moisture absorption and loss in eggs of Melanoplus bilituratus. Can. 7. Zool., 30, 55-82.

uvarov, B. 1966. Grasshoppers and Locusts. Cambridge University Press. 